PROCEEDINGS OF THE

AMERICAN MATHEMATICAL SOCIETY

Volume 137, Number 4, April 2009, Pages 1421-1429

S 0002-9939(08)09708-6

Article electronically published on October 29, 2008

\title{
ON THE TOPOLOGY OF POINTWISE CONVERGENCE ON THE BOUNDARIES OF $L_{1}$-PREDUALS
}

\author{
WARREN B. MOORS AND JIŘÍ SPURNÝ \\ (Communicated by Nigel J. Kalton)
}

\begin{abstract}
In this paper we prove a theorem more general than the following: "If $(X,\|\cdot\|)$ is an $L_{1}$-predual, $B$ is any boundary of $X$ and $\left\{x_{n}: n \in \mathbb{N}\right\}$ is any subset of $X$, then the closure of $\left\{x_{n}: n \in \mathbb{N}\right\}$ with respect to the topology of pointwise convergence on $B$ is separable with respect to the topology generated by the norm, whenever $\operatorname{Ext}\left(B_{X^{*}}\right)$ is weak* Lindelöf." Several applications of this result are also presented.
\end{abstract}

\section{INTRODUCTION}

We shall say that a Banach space $(X,\|\cdot\|)$ is an $L_{1}$-predual if $X^{*}$ is isometric to $L_{1}(\mu)$ for some suitable measure $\mu$. Some examples of $L_{1}$-preduals include $(C(K)$, $\left.\|\cdot\|_{\infty}\right)$, and more generally, the space of continuous affine functions on a Choquet simplex (see [10 for the definition) endowed with the supremum norm (see, 4, Proposition 3.23]). We shall also consider the notion of a boundary. Specifically, for a nontrivial Banach space $X$ over $\mathbb{R}$ we say that a subset $B$ of $B_{X^{*}}$, the closed unit ball of $X^{*}$, is a boundary if for each $x \in X$ there exists a $b^{*} \in B$ such that $b^{*}(x)=\|x\|$. The prototypical example of a boundary is $\operatorname{Ext}\left(B_{X^{*}}\right)$, the set of all extreme points of $B_{X^{*}}$, but there are many other interesting examples given in [9].

In the recent paper 9 ] the authors investigate the topology on a Banach space $X$ that is generated by $\operatorname{Ext}\left(B_{X^{*}}\right)$ and, more generally, the topology on $X$ generated by an arbitrary boundary of $X$. This paper continues this study.

To be more precise we must first introduce some notation. For a nonempty subset $Y$ of the dual of a Banach space $X$ we shall denote by $\sigma(X, Y)$ the weakest linear topology on $X$ that makes all the functionals from $Y$ continuous. In [9] the authors show (see, [9, Theorem 2.2]) using [3, Lemma 1] that for any compact Hausdorff space $K$, any countable subset $\left\{x_{n}: n \in \mathbb{N}\right\}$ of $C(K)$ and any boundary $B$ of $\left(C(K),\|\cdot\|_{\infty}\right)$, the closure of $\left\{x_{n}: n \in \mathbb{N}\right\}$ with respect to the $\sigma(C(K), B)$ topology is separable with respect to the topology generated by the norm. In this paper we extend this result by showing that if $(X,\|\cdot\|)$ is an $L_{1}$-predual, $B$ is any boundary of $X$ and $\left\{x_{n}: n \in \mathbb{N}\right\}$ is any subset of $X$, then the closure of $\left\{x_{n}: n \in \mathbb{N}\right\}$

Received by the editors May 22, 2008.

2000 Mathematics Subject Classification. Primary 46A50; Secondary 46B20.

Key words and phrases. Compact convex, extreme points, boundary, $L_{1}$-predual.

The second author was supported by the research project MSM 0021620839 financed by MSMT and by the grant GA ČR 201/07/0388. 
in the $\sigma(X, B)$ topology is separable with respect to the topology generated by the norm whenever $\operatorname{Ext}\left(B_{X^{*}}\right)$ is weak* Lindelöf.

We conclude this paper with some applications that indicate the utility of our results.

\section{Preliminary Results}

Let $X$ be a topological space and let $\mathcal{F}$ be a family of nonempty, closed and separable subsets of $X$. Then $\mathcal{F}$ is rich if the following two conditions are fulfilled:

(i) for every separable subspace $Y$ of $X$, there exists a $Z \in \mathcal{F}$ such that $Y \subseteq Z$;

(ii) for every increasing sequence $\left(Z_{n}: n \in \mathbb{N}\right)$ in $\mathcal{F}, \overline{\bigcup_{n \in \mathbb{N}} Z_{n}} \in \mathcal{F}$.

For any topological space $X$, the collection of all rich families of subsets forms a partially ordered set, under the binary relation of set inclusion. This partially ordered set has a greatest element, namely,

$$
\mathcal{G}_{X}:=\{S \subseteq X: S \text { is a nonempty, closed and separable subset of } X\} .
$$

On the other hand, if $X$ is a separable space, then the partially ordered set has a least element, namely, $\mathcal{G}_{\varnothing}:=\{X\}$.

The raison d'être for rich families is revealed next.

Proposition 1. Suppose that $X$ is a topological space. If $\left\{\mathcal{F}_{n}: n \in \mathbb{N}\right\}$ are rich families of $X$, then so is $\bigcap_{n \in \mathbb{N}} \mathcal{F}_{n}$.

For a proof of this proposition see [2, Proposition 1.1].

Throughout this paper we will be primarily working with Banach spaces, so a natural class of rich families, given a Banach space $X$, is the family of all closed separable linear subspaces of $X$, which we denote by $\mathcal{S}_{X}$. There are however many other interesting examples of rich families that can be found in [2] and [7.

For our first result we will provide another nontrivial example of a rich family, but to achieve this we first need a preliminary result that characterises when a given Banach space is an $L_{1}$-predual.

Lemma 1 ([, $\S 21$, Theorem 7]). For a Banach space $X$ the following are equivalent:

(i) $X$ is an $L_{1}$-predual;

(ii) for each weak* continuous convex function $f$ on $B_{X^{*}}$,

$$
f^{*}(0)=\frac{1}{2} \max \left\{f\left(x^{*}\right)+f\left(-x^{*}\right): x^{*} \in B_{X^{*}}\right\},
$$

where $f^{*}=\inf \left\{h: h \geq f\right.$ and $h$ is weak $*^{*}$ continuous and affine on $\left.B_{X^{*}}\right\}$.

Before proceeding further we shall introduce the following notation. If $X$ is a normed linear space, then each $x \in X$ defines a weak* continuous affine function $\widehat{x}$ on $B_{X^{*}}$ via the canonical embbeding, that is, $\widehat{x}\left(x^{*}\right):=x^{*}(x)$ for all $x^{*} \in B_{X^{*}}$.

Theorem 1. Let $X$ be an $L_{1}$-predual. Then the set of all closed separable linear subspaces of $X$ that are themselves $L_{1}$-preduals forms a rich family.

Proof. Let $\mathscr{L}:=\left\{Z \in \mathcal{S}_{X}: Z\right.$ is an $L_{1}$-predual $\}$. We shall verify that $\mathscr{L}$ is a rich family. So first let us consider an arbitrary separable closed linear subspace $Y$ of $X$. Then by [6, $\S 23$, Lemma 1] there exists a closed separable subspace $Z \in \mathscr{L}$ such that $Y \subseteq Z$. Next, let us consider an increasing sequence $\left(Z_{n}: n \in \mathbb{N}\right)$ in $\mathscr{L}$ 
and let $Z:=\overline{\bigcup_{n \in \mathbb{N}} Z_{n}}$. To show that $Z \in \mathscr{L}$ we shall appeal to Lemma 1, Let $f$ be a weak* continuous convex function on $B_{Z^{*}}$. Since

$$
\frac{1}{2} \max \left\{f\left(x^{*}\right)+f\left(-x^{*}\right): x^{*} \in B_{Z^{*}}\right\} \leq f^{*}(0),
$$

it is enough to verify that for each $\varepsilon>0, f^{*}(0) \leq \frac{1}{2} \max \left\{f\left(x^{*}\right)+f\left(-x^{*}\right): x^{*} \in\right.$ $\left.B_{Z^{*}}\right\}+\varepsilon$. To this end, suppose that $\varepsilon>0$. Since $f$ is weak* continuous and convex and $B_{Z^{*}}$ is weak* compact, by [1, Corollary I.1.3] there exist $z_{i} \in Z$ and $c_{i} \in \mathbb{R}$, $i=1, \ldots, n$, such that the weak ${ }^{*}$ convex continuous $g: B_{Z^{*}} \rightarrow \mathbb{R}$ defined by

$$
g:=\max \left\{\widehat{z_{1}}+c_{1}, \widehat{z_{2}}+c_{2}, \ldots, \widehat{z_{n}}+c_{n}\right\}
$$

satisfies

$$
f\left(z^{*}\right)-\varepsilon<g\left(z^{*}\right)<f\left(z^{*}\right), \quad z^{*} \in B_{Z^{*}} .
$$

Since $\bigcup_{n \in \mathbb{N}} Z_{n}$ is dense in $Z$ we may further assume that all the elements $z_{i}$ are contained in some fixed $Z_{j}, j \in \mathbb{N}$.

Next, let $r: B_{Z^{*}} \rightarrow B_{Z_{j}^{*}}$ be the restriction mapping (i.e., $r\left(z^{*}\right)=\left.z^{*}\right|_{Z_{j}}$ for all $\left.z^{*} \in B_{Z^{*}}\right)$ and let $h: B_{Z_{j}^{*}} \rightarrow \mathbb{R}$ be defined by $h:=\max \left\{\widehat{z_{1}}+c_{1}, \widehat{z_{2}}+c_{2}, \ldots, \widehat{z_{n}}+c_{n}\right\}$. Then $h$ is weak* continuous and convex on $B_{Z_{j}^{*}}$ and $g=h \circ r$. Moreover, by the definition of $g$ (and the fact that $r$ is weak ${ }^{*}$-to-weak* continuous and linear) we have that $g^{*}\left(z^{*}\right) \leq h^{*}\left(r\left(z^{*}\right)\right)$ for all $z^{*} \in B_{Z^{*}}$. Now, by the assumption that $Z_{j}$ is an $L_{1}$-predual (and Lemma 1 ) there exists a $y^{*} \in B_{Z_{j}^{*}}$ such that

$$
h^{*}(0)=\frac{1}{2}\left[h\left(y^{*}\right)+h\left(-y^{*}\right)\right] \text {. }
$$

Choose $z^{*} \in r^{-1}\left(y^{*}\right)$, which is nonempty by the Hahn-Banach extension theorem. Then,

$$
g^{*}(0) \leq h^{*}(0)=\frac{1}{2}\left[h\left(y^{*}\right)+h\left(-y^{*}\right)\right]=\frac{1}{2}\left[g\left(z^{*}\right)+g\left(-z^{*}\right)\right] \leq g^{*}(0) .
$$

Therefore,

$$
\begin{aligned}
f^{*}(0)-\varepsilon=(f-\varepsilon)^{*}(0) & \leq g^{*}(0)=\frac{1}{2}\left[g\left(z^{*}\right)+g\left(-z^{*}\right)\right] \\
& \leq \frac{1}{2}\left[f\left(z^{*}\right)+f\left(-z^{*}\right)\right] \\
& \leq \frac{1}{2} \max \left\{f\left(x^{*}\right)+f\left(-x^{*}\right): x^{*} \in B_{Z^{*}}\right\} .
\end{aligned}
$$

That is, $f^{*}(0) \leq \frac{1}{2} \max \left\{f\left(x^{*}\right)+f\left(-x^{*}\right): x^{*} \in B_{Z^{*}}\right\}+\varepsilon$, which completes the proof.

Before we can introduce another class of rich families, we require the following lemma, which is a Banach space version of [11, Theorem 2.10].

Lemma 2. Let $Y$ be a closed separable linear subspace of a Banach space $X$ and suppose that $L \subseteq \operatorname{Ext}\left(B_{X^{*}}\right)$ is weak $k^{*}$ Lindelöf. Then there exists a closed separable linear subspace $Z$ of $X$, containing $Y$, such that for any $l^{*} \in L$ and any $x^{*}, y^{*} \in$ $B_{Z^{*}}$, if $\left.l^{*}\right|_{Z}=\frac{1}{2}\left(x^{*}+y^{*}\right)$, then $\left.x^{*}\right|_{Y}=\left.y^{*}\right|_{Y}$.

Proof. Let $\mathscr{B}$ be a countable base for the topology on $\left(B_{Y^{*}}\right.$, weak* $)$ consisting of closed convex sets. Recall that such a base exists because $\left(B_{Y^{*}}\right.$, weak $\left.{ }^{*}\right)$ is compact, by the Banach-Alaoglu Theorem, and $\left(B_{Y^{*}}\right.$, weak* $\left.{ }^{*}\right)$ is metrizable, since $Y$ is separable. Let: 
(i) $\mathscr{F}:=\left\{r^{-1}(B): B \in \mathscr{B}\right\}$, where $r: B_{X^{*}} \rightarrow B_{Y^{*}}$ is the restriction mapping;

(ii) $\mathscr{R}:=\left\{\frac{1}{2}\left(F_{1}+F_{2}\right): F_{1}, F_{2} \in \mathscr{F}\right.$ and $\left.F_{1} \cap F_{2}=\varnothing\right\}$.

By construction $\bigcup \mathscr{R} \subseteq B_{X^{*}} \backslash \operatorname{Ext}\left(B_{X^{*}}\right)$ and so $L \cap \bigcup \mathscr{R}=\varnothing$. Furthermore, for each $l^{*} \in L$ and $F \in \mathscr{R}$ there exists a $y \in X$ such that

$$
\sup \left\{\widehat{y}\left(f^{*}\right): f^{*} \in F\right\}<\widehat{y}\left(l^{*}\right) .
$$

Therefore, since $L$ is weak* Lindelöf for each $F \in \mathscr{R}$ there exists a countable subset $C_{F}$ in $X$ such that, for each $l^{*} \in L$ there exists a $y \in C_{F}$ such that $\sup \left\{\widehat{y}\left(f^{*}\right)\right.$ : $\left.f^{*} \in F\right\}<\widehat{y}\left(l^{*}\right)$. If we set $C:=\bigcup\left\{C_{F}: F \in \mathscr{R}\right\}$ and $Z:=\overline{\operatorname{span}}(C \cup X)$, then $X \subseteq Z$ and $Z$ is a closed separable linear subspace of $X$.

It now only remains to verify that if $l^{*} \in L, x^{*}, y^{*} \in B_{Z^{*}}$ and $\left.l^{*}\right|_{Z}=\frac{1}{2}\left(x^{*}+y^{*}\right)$, then $\left.x^{*}\right|_{Y}=\left.y^{*}\right|_{Y}$. So, in order to obtain a contradiction, suppose that for some $l^{*} \in L$ and $x^{*}, y^{*} \in B_{Z^{*}},\left.l^{*}\right|_{Z}=\frac{1}{2}\left(x^{*}+y^{*}\right)$ but $\left.x^{*}\right|_{Y} \neq\left. y^{*}\right|_{Y}$. Then there exists $B_{1}, B_{2} \in \mathscr{B}$ such that $\left.x^{*}\right|_{Y} \in B_{1}$ and $\left.y^{*}\right|_{Y} \in B_{2}$ and $B_{1} \cap B_{2}=\varnothing$. Set $F_{1}:=r^{-1}\left(B_{1}\right)$ and $F_{2}:=r^{-1}\left(B_{2}\right)$. Then $F_{1}, F_{2} \in \mathscr{F}$ and $F_{1} \cap F_{2}=\varnothing$. Now, by the Hahn-Banach Extension Theorem there exist $x_{1}^{*} \in B_{X^{*}}$ and $y_{1}^{*} \in B_{X^{*}}$ such that $\left.x_{1}^{*}\right|_{Z}=x^{*}$ and $\left.y_{1}^{*}\right|_{Z}=y^{*}$. Moreover,

$$
\left.x_{1}^{*}\right|_{Y}=\left.\left(\left.x_{1}^{*}\right|_{Z}\right)\right|_{Y}=\left.x^{*}\right|_{Y} \in B_{1} \quad \text { and }\left.\quad y_{1}^{*}\right|_{Y}=\left.\left(\left.y_{1}^{*}\right|_{Z}\right)\right|_{Y}=\left.y^{*}\right|_{Y} \in B_{2} \text {. }
$$

That is, $x_{1}^{*} \in F_{1}$ and $y_{1}^{*} \in F_{2}$. Therefore, $\frac{1}{2}\left(x_{1}^{*}+y_{1}^{*}\right) \in \frac{1}{2}\left(F_{1}+F_{2}\right)=: F$. Since $F \in \mathscr{R}$, by the construction there exists a $y \in C_{F} \subseteq C \subseteq Z$ such that $\sup \left\{\widehat{y}\left(f^{*}\right)\right.$ : $\left.f^{*} \in F\right\}<\widehat{y}\left(l^{*}\right)$. In particular,

$$
\frac{1}{2}\left(x^{*}+y^{*}\right)(y)=\widehat{y}\left(\frac{1}{2}\left(x_{1}^{*}+y_{1}^{*}\right)\right)<l^{*}(y)=\left(\left.l^{*}\right|_{Z}\right)(y) .
$$

However, this contradicts the fact that $\frac{1}{2}\left(x^{*}+y^{*}\right)=\left.l^{*}\right|_{Z}$.

Theorem 2. Let $X$ be a Banach space and let $L \subseteq \operatorname{Ext}\left(B_{X^{*}}\right)$ be a weak $k^{*}$ Lindelöf subset. Then the set of all $Z$ in $\mathcal{S}_{X}$ such that $\left\{\left.l^{*}\right|_{Z}: l^{*} \in L\right\} \subseteq \operatorname{Ext}\left(B_{Z^{*}}\right)$ forms a rich family.

Proof. Let $\mathscr{L}$ denote the family of all closed separable linear subspaces $Z$ of $X$ such that $\left\{\left.l^{*}\right|_{Z}: l^{*} \in L\right\} \subseteq \operatorname{Ext}\left(B_{Z^{*}}\right)$. We shall verify that $\mathscr{L}$ is a rich family of closed separable linear subspaces of $X$. So first let us consider an arbitrary closed separable linear subspace $Y$ of $X$, with the aim of showing that there exists a subspace $Z \in \mathscr{L}$ such that $Y \subseteq Z$. We begin by inductively applying Lemma 2 to obtain an increasing sequence $\left(Z_{n}: n \in \mathbb{N}\right)$ of closed separable linear subspaces of $X$ such that: $Y \subseteq Z_{1}$ and for any $l^{*} \in L$ and any $x^{*}, y^{*} \in B_{Z_{n+1}^{*}}$, if $\left.l^{*}\right|_{Z_{n+1}}=\frac{1}{2}\left(x^{*}+y^{*}\right)$, then $\left.x^{*}\right|_{Z_{n}}=\left.y^{*}\right|_{Z_{n}}$.

We now claim that if $Z:=\overline{\bigcup_{n \in \mathbb{N}} Z_{n}}$, then $\left.l^{*}\right|_{Z} \in \operatorname{Ext}\left(B_{Z^{*}}\right)$ for each $l^{*} \in L$. To this end, suppose that $l^{*} \in L$ and $\left.l^{*}\right|_{Z}=\frac{1}{2}\left(x^{*}+y^{*}\right)$ for some $x^{*}, y^{*} \in B_{Z^{*}}$. Then for each $n \in \mathbb{N}$,

$$
\left.l^{*}\right|_{Z_{n+1}}=\left.\left(\left.l^{*}\right|_{Z}\right)\right|_{Z_{n+1}}=\left.\frac{1}{2}\left(x^{*}+y^{*}\right)\right|_{Z_{n+1}}=\frac{1}{2}\left(\left.x^{*}\right|_{Z_{n+1}}+\left.y^{*}\right|_{Z_{n+1}}\right)
$$

and $\left.x^{*}\right|_{Z_{n+1}},\left.y^{*}\right|_{Z_{n+1}} \in B_{Z_{n+1}^{*}}$ Therefore, by construction, $\left.x^{*}\right|_{Z_{n}}=\left.y^{*}\right|_{Z_{n}}$. Now since $\bigcup_{n \in \mathbb{N}} Z_{n}$ is dense in $Z$ and both $x^{*}$ and $y^{*}$ are continuous, we may deduce that $x^{*}=y^{*}$, which in turn implies that $\left.l^{*}\right|_{Z} \in \operatorname{Ext}\left(B_{Z^{*}}\right)$. This shows that $Y \subseteq Z$ and $Z \in \mathscr{L}$. 
To complete this proof we must verify that for each increasing sequence of closed separable subspaces $\left(Z_{n}: n \in \mathbb{N}\right)$ in $\mathscr{L}, \overline{\bigcup_{n \in \mathbb{N}} Z_{n}} \in \mathscr{L}$. This, however, follows easily from the definition of the family $\mathscr{L}$.

Let $X$ be a normed linear space. Then we say that an element $x^{*} \in B_{X^{*}}$ is weak $k^{*}$ exposed if there exists an element $x \in X$ such that $y^{*}(x)<x^{*}(x)$ for all $y^{*} \in B_{X^{*}} \backslash\left\{x^{*}\right\}$. It is not difficult to show that if $\operatorname{Exp}\left(B_{X^{*}}\right)$ denotes the set of all weak* exposed points of $B_{X^{*}}$, then $\operatorname{Exp}\left(B_{X^{*}}\right) \subseteq \operatorname{Ext}\left(B_{X^{*}}\right)$. However, if $X$ is a separable $L_{1}$-predual, then the relationship between $\operatorname{Exp}\left(B_{X^{*}}\right)$ and $\operatorname{Ext}\left(B_{X^{*}}\right)$ is much closer.

Lemma 3 (13, Lemma 3.3(b)]). If $X$ is a separable $L_{1}$-predual, then $\operatorname{Exp}\left(B_{X^{*}}\right)=$ $\operatorname{Ext}\left(B_{X^{*}}\right)$.

Let us also pause for a moment to recall that if $B$ is any boundary of a Banach space $X$, then

$$
\operatorname{Exp}\left(B_{X^{*}}\right) \subseteq B \cap \operatorname{Ext}\left(B_{X^{*}}\right) \subseteq \operatorname{Ext}\left(B_{X^{*}}\right) \subseteq \bar{B}^{\text {weak }^{*}} .
$$

The fact that $\operatorname{Ext}\left(B_{X^{*}}\right) \subseteq \bar{B}^{\text {weak* }}$ follows from Milman's theorem, [10, page 8] and the fact that $B_{X^{*}}=\overline{\mathrm{co}}^{\text {weak }^{*}}(B)$, which in turn follows from a separation argument. Let us also take this opportunity to observe that if $B_{X}$ denotes the closed unit ball in $X$, then $B_{X}$ is closed in the $\sigma(X, B)$ topology for any boundary $B$ of $X$. Finally, let us end this section with one more simple observation that will turn out to be useful in our later endeavours.

Proposition 2. Suppose that $Y$ is a linear subspace of a Banach space $(X,\|\cdot\|)$ and $B$ is any boundary for $X$. Then for each $e^{*} \in \operatorname{Exp}\left(B_{Y^{*}}\right)$ there exists $b^{*} \in B$ such that $e^{*}=\left.b^{*}\right|_{Y}$.

Proof. Suppose that $e^{*} \in \operatorname{Exp}\left(B_{Y^{*}}\right)$. Then there exists an $x \in Y$ such that $y^{*}(x)<$ $e^{*}(x)$ for each $y^{*} \in B_{Y^{*}} \backslash\left\{e^{*}\right\}$. By the fact that $B$ is a boundary of $(X,\|\cdot\|)$ there exists a $b^{*} \in B$ such that $b^{*}(x)=\|x\| \neq 0$. Then for any $y^{*} \in B_{Y^{*}}$ we have

$$
y^{*}(x) \leq\left|y^{*}(x)\right| \leq\left\|y^{*}\right\|\|x\| \leq\|x\|=b^{*}(x)=\left(\left.b^{*}\right|_{Y}\right)(x) .
$$

In particular, $e^{*}(x) \leq\left. b^{*}\right|_{Y}(x)$. Since $\left.b^{*}\right|_{Y} \in B_{Y^{*}}$ and $y^{*}(x)<e^{*}(x)$ for all $y^{*} \in$ $B_{Y^{*}} \backslash\left\{e^{*}\right\}$, it must be the case that $e^{*}=\left.b^{*}\right|_{Y}$.

This ends our preliminary section.

\section{The MAIN RESUlts}

Theorem 3. Let $B$ be any boundary for a Banach space $X$ that is an $L_{1}$-predual and suppose that $\left\{x_{n}: n \in \mathbb{N}\right\} \subseteq X$. Then

$$
{\overline{\left\{x_{n}: n \in \mathbb{N}\right\}}}^{\sigma(X, B)} \subseteq{\overline{\left\{x_{n}: n \in \mathbb{N}\right\}}}^{\sigma\left(X, \operatorname{Ext}\left(B_{X^{*}}\right)\right)} .
$$

Proof. In order to obtain a contradiction let us suppose that

$$
{\overline{\left\{x_{n}: n \in \mathbb{N}\right\}}}^{\sigma(X, B)} \nsubseteq{\overline{\left\{x_{n}: n \in \mathbb{N}\right\}}}^{\sigma\left(X, \operatorname{Ext}\left(B_{X^{*}}\right)\right)} .
$$

Choose $x \in{\overline{\left\{x_{n}: n \in \mathbb{N}\right\}}}^{\sigma(X, B)} \backslash{\overline{\left\{x_{n}: n \in \mathbb{N}\right\}}}^{\sigma\left(X, \operatorname{Ext}\left(B_{X^{*}}\right)\right)}$. Then there exists a finite set $\left\{e_{1}^{*}, e_{2}^{*}, \ldots, e_{m}^{*}\right\} \subseteq \operatorname{Ext}\left(B_{X^{*}}\right)$ and $\varepsilon>0$ so that

$$
\left\{y \in X:\left|e_{k}^{*}(x)-e_{k}^{*}(y)\right|<\varepsilon \text { for all } 1 \leq k \leq m\right\} \cap\left\{x_{n}: n \in \mathbb{N}\right\}=\varnothing .
$$


Let $Y:=\overline{\operatorname{span}}\left(\left\{x_{n}: n \in \mathbb{N}\right\} \cup\{x\}\right)$, let $\mathcal{F}_{1}$ be any rich family of $L_{1}$-preduals whose existence is guaranteed by Theorem 1 , and let $\mathcal{F}_{2}$ be any rich family such that for every $Z \in \mathcal{F}_{2}$ and every $1 \leq k \leq m,\left.e_{k}^{*}\right|_{Z} \in \operatorname{Ext}\left(B_{Z^{*}}\right)$, whose existence is guaranteed by Theorem 2. Next, let us choose $Z \in \mathcal{F}_{1} \cap \mathcal{F}_{2}$ so that $Y \subseteq Z$. Recall that this is possible because, by Proposition $1, \mathcal{F}_{1} \cap \mathcal{F}_{2}$ is a rich family. Since $Z$ is a separable $L_{1}$-predual we have by Lemma 3 that $\left.e_{k}^{*}\right|_{Z} \in \operatorname{Exp}\left(B_{Z^{*}}\right)$ for each $1 \leq k \leq m$. Now, by Proposition 2 , for each $1 \leq k \leq m$ there exists a $b_{k}^{*} \in B$ such that $\left.e_{k}^{*}\right|_{Z}=\left.b_{k}^{*}\right|_{Z}$. Therefore,

$$
\begin{aligned}
\left|b_{k}^{*}(x)-b_{k}^{*}\left(x_{j}\right)\right| & =\left|\left(\left.b_{k}^{*}\right|_{Z}\right)(x)-\left(\left.b_{k}^{*}\right|_{Z}\right)\left(x_{j}\right)\right| \\
& =\left|\left(\left.e_{k}^{*}\right|_{Z}\right)(x)-\left(\left.e_{k}^{*}\right|_{Z}\right)\left(x_{j}\right)\right|=\left|e_{k}^{*}(x)-e_{k}^{*}\left(x_{j}\right)\right|
\end{aligned}
$$

for all $j \in \mathbb{N}$ and all $1 \leq k \leq m$. Thus,

$$
\left\{y \in X:\left|b_{k}^{*}(x)-b_{k}^{*}(y)\right|<\varepsilon \text { for all } 1 \leq k \leq m\right\} \cap\left\{x_{n}: n \in \mathbb{N}\right\}=\varnothing .
$$

This contradicts the fact that $x \in{\overline{\left\{x_{n}: n \in \mathbb{N}\right\}}}^{\sigma(X, B)}$, which completes the proof.

Corollary 1 ([13, Theorem 1.1(a)]). Let $B$ be any boundary for a Banach space $X$ that is an $L_{1}$-predual. Then every relatively countably $\sigma(X, B)$-compact subset is relatively countably $\sigma\left(X, \operatorname{Ext}\left(B_{X^{*}}\right)\right)$-compact. In particular, every norm bounded, relatively countably $\sigma(X, B)$-compact subset is relatively weakly compact.

Proof. Suppose that a nonempty set $C \subseteq X$ is relatively countably $\sigma(X, B)$ compact. Let $\left\{c_{n}: n \in \mathbb{N}\right\}$ be any sequence in $C$. Then by Theorem 3

$$
\varnothing \neq \bigcap_{n \in \mathbb{N}}{\overline{\left\{c_{k}: k \geq n\right\}}}^{\sigma(X, B)} \subseteq \bigcap_{n \in \mathbb{N}}{\overline{\left\{c_{k}: k \geq n\right\}}}^{\sigma\left(X, \operatorname{Ext}\left(B_{X^{*}}\right)\right)} .
$$

Hence $C$ is relatively countably $\sigma\left(X, \operatorname{Ext}\left(B_{X^{*}}\right)\right)$-compact. In the case when $C$ is also norm bounded the result follows from either [5] or 8 .

Recall that a network for a topological space $X$ is a family $\mathscr{N}$ of subsets of $X$ such that for any point $x \in X$ and any open neighbourhood $U$ of $x$ there is an $N \in \mathscr{N}$ such that $x \in N \subseteq U$, and a topological space $X$ is said to be $\aleph_{0}$-monolithic if the closure of every countable set has a countable network.

The next corollary generalises [9, Theorem 2.2].

Corollary 2. Let $B$ be any boundary for a Banach space $X$ that is an $L_{1}$-predual and suppose that $\left\{x_{n}: n \in \mathbb{N}\right\} \subseteq X$. Then ${\overline{\left\{x_{n}: n \in \mathbb{N}\right\}}}^{\sigma(X, B)}$ is norm separable whenever $X$ is $\aleph_{0}$-monolithic in the $\sigma\left(X, \operatorname{Ext}\left(B_{X^{*}}\right)\right)$ topology. In particular, ${\overline{\left\{x_{n}: n \in \mathbb{N}^{\sigma}\right.}}^{\sigma(X, B)}$ is norm separable whenever $\operatorname{Ext}\left(B_{X^{*}}\right)$ is weak $k^{*}$ Lindelöf.

Proof. From Theorem 3, ${\overline{\left\{x_{n}: n \in \mathbb{N}\right\}}}^{\sigma(X, B)} \subseteq{\overline{\left\{x_{n}: n \in \mathbb{N}\right\}}}^{\sigma\left(X, \operatorname{Ext}\left(B_{X^{*}}\right)\right)}$. Since $X$ is $\aleph_{0}$-monolithic in the $\sigma\left(X, \operatorname{Ext}\left(B_{X^{*}}\right)\right)$ topology ${\overline{\left\{x_{n}: n \in \mathbb{N}\right\}}}^{\sigma\left(X, \operatorname{Ext}\left(B_{X^{*}}\right)\right)}$ has a countable network with respect to the $\sigma\left(X, \operatorname{Ext}\left(B_{X^{*}}\right)\right)$ topology and hence so does ${\overline{\left\{x_{n}: n \in \mathbb{N}\right\}}}^{\sigma(X, B)}$. Let $\mathcal{A}\left(B_{X^{*}}\right)$ denote the set of all weak* continuous real-valued affine mappings on $B_{X^{*}}$. Then the mapping $T:(X,\|\cdot\|) \rightarrow\left(\mathcal{A}\left(B_{X^{*}}\right),\|\cdot\|_{\infty}\right)$ defined by $T(x)\left(x^{*}\right):=x^{*}(x)$ for all $x^{*} \in B_{X^{*}}$ is a homeomorphic embedding with respect to both (i) the norm topologies on $X$ and $\mathcal{A}\left(B_{X^{*}}\right)$ and (ii) the $\sigma\left(X, \operatorname{Ext}\left(X^{*}\right)\right)$ and $\tau_{p}\left(\operatorname{Ext}\left(B_{X^{*}}\right)\right)$ topologies on $X$ and $\mathcal{A}\left(B_{X^{*}}\right)$ respectively. The result then follows from [9, Theorem 2.6]. The last claim follows from [9, Theorem 2.14], where 
it is shown that if $\operatorname{Ext}\left(B_{X^{*}}\right)$ is weak* Lindelöf, then $X$ is $\aleph_{0}$-monolithic in the $\sigma\left(X, \operatorname{Ext}\left(B_{X^{*}}\right)\right)$ topology.

In [9] many conditions are given under which $\sigma\left(X, \operatorname{Ext}\left(B_{X^{*}}\right)\right)$ is $\aleph_{0}$-monolithic.

To demonstrate how this last theorem may be applied we shall present some sample applications.

\section{Applications}

Our first application is to metrizability of compact convex sets. If $K$ is a compact convex set in a real locally convex space, let $\mathcal{A}(K)$ stand for the space of all affine continuous functions on $K$.

Proposition 3. Let $K$ be a Choquet simplex in a separated locally convex space (over $\mathbb{R}$ ) such that every regular Borel probability measure carried on $\operatorname{Ext}(K)$ is atomic. Then $K$ is metrizable if, and only if, the space $\left(B_{\mathcal{A}(K)}, \sigma(\mathcal{A}(K), B)\right)$ is separable, for some boundary $B$ of $\left(\mathcal{A}(K),\|\cdot\|_{\infty}\right)$.

Proof. This follows directly from Theorem 3 and [9, Theorem 2.19].

We remark that there exists a nonmetrizable Choquet simplex $K$ and a boundary $B$ of $\left(\mathcal{A}(K),\|\cdot\|_{\infty}\right)$ such that $\left(B_{\mathcal{A}(K)}, \sigma(A(K), B)\right)$ is separable. (It is shown in 13, Section 4] that the construction of [9, Example 2.10] yields the required example.)

Our final few results concern automatic continuity. In particular, the next result improves [12, Theorem 6].

Proposition 4. Let $B$ be any boundary for a Banach space $X$ that is an $L_{1}$-predual and suppose that $A$ is a a separable Baire space. If $X$ is $\aleph_{0}$-monolithic in the $\sigma\left(X, \operatorname{Ext}\left(B_{X^{*}}\right)\right)$ topology, then for each continuous mapping $f: A \rightarrow(X, \sigma(X, B))$ there exists a dense subset $D$ of $A$ such that $f$ is continuous with respect to the norm topology on $X$ at each point of $D$.

Proof. Fix $\varepsilon>0$ and consider the open set:

$$
O_{\varepsilon}:=\bigcup\{U \subseteq A: U \text { is open and }\|\cdot\|-\operatorname{diam}[f(U)] \leq 2 \varepsilon\} .
$$

We shall show that $O_{\varepsilon}$ is dense in $A$. To this end, let $W$ be a nonempty open subset of $A$ and let $\left\{a_{n}: n \in \mathbb{N}\right\}$ be a countable dense subset of $W$. Then by continuity

$$
f(W) \subseteq{\overline{\left\{f\left(a_{n}\right): n \in \mathbb{N}\right\}}}^{\sigma(X, B)},
$$

which is norm separable by Corollary 2. Therefore there exists a countable set $\left\{x_{n}: n \in \mathbb{N}\right\}$ in $X$ such that $f(W) \subseteq \bigcup_{n \in \mathbb{N}}\left(x_{n}+\varepsilon B_{X}\right)$. For each $n \in \mathbb{N}$, let $C_{n}:=f^{-1}\left(x_{n}+\varepsilon B_{X}\right)$. Since each $x_{n}+\varepsilon B_{X}$ is closed in the $\sigma(X, B)$ topology each set $C_{n}$ is closed in $A$ and, moreover, $W \subseteq \bigcup_{n \in \mathbb{N}} C_{n}$. Since $W$ is of the second Baire category in $A$ there exist a nonempty open set $U \subseteq W$ and a $k \in \mathbb{N}$ such that $U \subseteq C_{k}$. Then $U \subseteq O_{\varepsilon} \cap W$ and $O_{\varepsilon}$ is indeed dense in $A$. Hence $f$ is $\|\cdot\|$-continuous at each point of $\bigcap_{n \in \mathbb{N}} O_{1 / n}$.

Theorem 4. Suppose that $A$ is a topological space with countable tightness that possesses a rich family $\mathcal{F}$ of Baire subspaces and suppose that $X$ is an $L_{1}$-predual. Then for any boundary $B$ of $X$ and any continuous function $f: A \rightarrow(X, \sigma(X, B))$ there exists a dense subset $D$ of $A$ such that $f$ is continuous with respect to the norm topology on $X$ at each point of $D$ provided $X$ is $\aleph_{0}$-monolithic in the $\sigma\left(X, \operatorname{Ext}\left(B_{X^{*}}\right)\right)$ topology. 
Proof. In order to obtain a contradiction let us suppose that $f$ does not have a dense set of points of continuity with respect to the norm topology on $X$. Since $A$ is a Baire space (by [7, Theorem 3.3]), this implies that for some $\varepsilon>0$ the open set

$$
O_{\varepsilon}:=\bigcup\{U \subseteq A: U \text { is open and }\|\cdot\|-\operatorname{diam}[f(U)] \leq 2 \varepsilon\}
$$

is not dense in $A$. That is, there exists a nonempty open subset $W$ of $A$ such that $W \cap O_{\varepsilon}=\varnothing$. For each $x \in A$, let $F_{x}:=\{y \in A:\|f(y)-f(x)\|>\varepsilon\}$. Then $x \in \overline{F_{x}}$ for each $x \in W$. Moreover, since $A$ has countable tightness, for each $x \in W$, there exists a countable subset $C_{x}$ of $F_{x}$ such that $x \in \overline{C_{x}}$.

Next, we inductively define an increasing sequence of separable subspaces $\left(F_{n}\right.$ : $n \in \mathbb{N})$ of $A$ and countable sets $\left(D_{n}: n \in \mathbb{N}\right)$ in $A$ such that:

(i) $W \cap F_{1} \neq \varnothing$;

(ii) $\bigcup\left\{C_{x}: x \in D_{n} \cap W\right\} \cup F_{n} \subseteq F_{n+1} \in \mathcal{F}$ for all $n \in \mathbb{N}$, where $D_{n}$ is any countable dense subset of $F_{n}$.

Note that since the family $\mathcal{F}$ is rich, this construction is possible.

Let $F:=\overline{\bigcup_{n \in \mathbb{N}} F_{n}}$ and $D:=\bigcup_{n \in \mathbb{N}} D_{n}$. Then $\bar{D}=F \in \mathcal{F}$ and $\|\cdot\|$-diam $[f(U)] \geq$ $\varepsilon$ for every nonempty open subset $U$ of $F \cap W$. Therefore, $\left.f\right|_{F}$ has no points of continuity in $F \cap W$ with respect to the $\|\cdot\|$-topology. This, however, contradicts Proposition 4.

Our final result improves [7, Theorem 4.7].

Corollary 3. Suppose that $A$ is a topological space with countable tightness that possesses a rich family of Baire subspaces and suppose that $K$ is a compact Hausdorff space. Then for any boundary of $\left(C(K),\|\cdot\|_{\infty}\right)$ and any continuous function $f: A \rightarrow(C(K), \sigma(C(K), B))$ there exists a dense subset $D$ of $A$ such that $f$ is continuous with respect to the $\|\cdot\|_{\infty}$-topology at each point of $D$.

\section{REFERENCES}

[1] Erik M. Alfsen, Compact convex sets and boundary integrals, Ergebnisse der Mathematik und ihrer Grenzgebiete, Band 57, Springer-Verlag, New York-Heidelberg, 1971. MR0445271 $(56: 3615)$

[2] Jonathan M. Borwein and Warren B. Moors, Separable determination of integrability and minimality of the Clarke subdifferential mapping, Proc. Amer. Math. Soc. 128 (2000), 215221. MR.1622793 (2000c:49025)

[3] Bernardo Cascales and Gilles Godefroy, Angelicity and the boundary problem, Mathematika 45 (1998), 105-112. MR1644346 (99f:46019)

[4] V. P. Fonf, J. Lindenstrauss and R. R. Phelps, Infinite dimensional convexity, Handbook of the geometry of Banach spaces, Vol. I, North-Holland, Amsterdam, 2001, 599-670. MR.1863703 (2003c:46014)

[5] S. S. Khurana, Pointwise compactness on extreme points, Proc. Amer. Math. Soc. 83 (1981), 347-348. MR624928 (82k:46018)

[6] H. E. Lacey, The isometric theory of classical Banach spaces, Die Grundlehren der Mathematischen Wissenschaften, Band 208, Springer-Verlag, New York-Heidelberg, 1974. MR 0493279 $(58: 12308)$

[7] Peijie Lin and Warren B. Moors, Rich families and the product of Baire spaces, Math. Balkanica, to appear, available at http://www.math.auckland.ac.nz/ moors/.

[8] Warren B. Moors, A characterisation of weak compactness in Banach spaces, Bull. Austral. Math. Soc. 55 (1997), 497-501. MR1456278 (98g:46019)

[9] Warren B. Moors and Evgenii A. Reznichenko, Separable subspaces of affine function spaces on compact convex sets, Topology Appl. 155 (2008), 1306-1322.

[10] R. Phelps, Lectures on Choquet's Theorem, second edition, Lecture Notes in Mathematics, no. 1757, Springer-Verlag, Berlin, 2001. MR1835574 (2002k:46001) 
[11] Evgenii A. Reznichenko, Compact convex spaces and their maps, Topology Appl. 36 (1990), 117-141. MR1068165 (91g:54010)

[12] J. Saint Raymond, Jeux topologiques et espaces de Namioka, Proc. Amer. Math. Soc. 87 (1983), 449-504. MR684646 (83m:54060)

[13] Jiří Spurný, The boundary problem for $L_{1}$-preduals. Illinois J. Math. to appear in 2009, available at http://www.karlin.mff.cuni.cz/kma-preprints/.

Department of Mathematics, The University of Auckland, Private Bag 92019, AuckLAND, NEW ZEALAND

E-mail address: moors@math.auckland.ac.nz

Faculty of Mathematics and Physics, Charles University, Sokolovská 83, 18675 Praha 8, Czech Republic

E-mail address: spurny@karlin.mff.cuni.cz 